\title{
Host galaxies of jetted narrow-line Seyfert 1 galaxies
}

\author{
Emilia Järvelä ${ }^{\sharp a b}$, Anne Lähteenmäki ${ }^{a b}$ and Marco Berton ${ }^{c d}$ \\ ${ }^{a}$ Aalto University Metsähovi Radio Observatory \\ Metsähovintie 114, FI-02540 Kylmälä, Finland \\ ${ }^{b}$ Aalto University Department of Electronics and Nanoengineering \\ P.O. Box 15500, FI-00076 AALTO, Finland \\ c Dipartimento di Fisica e Astronomia "G. Galilei", Università degli Studi di Padova \\ Vicolo dell'Osservatorio 3, 35122, Padova (Italy) \\ ${ }^{d}$ INAF - Osservatorio Astronomico di Brera \\ Via E. Bianchi 46, 23807, Merate (Italy) \\ E-mail: emilia.jarvela@aalto.fi
}

\begin{abstract}
We performed $J$-band observations of the host galaxies of nine narrow-line Seyfert 1 galaxies (NLS1). Seven of these sources have been detected at $37 \mathrm{GHz}$ and are likely to host relativistic jets. Host galaxy properties of the jetted NLS1 galaxies are not yet well known, and investigating them is essential to get more insight into their characteristics and the possibly heterogeneous nature of the NLS1 population. It also helps us to understand the overall evolution of NLS1 galaxies and how do they fit into the big picture of active galactic nuclei (AGN). We used the 2D photometric image decomposition algorithm GALFIT to model the morphologies of the hosts. We were able to reliably model five of the nine host galaxies, three of which have also been detected at $37 \mathrm{GHz}$. All five host galaxies are late-type, four of them host pseudobulges, and four are barred. A surprisingly large fraction, three out of five, show signs of interaction. These results support the idea that spiral galaxies with pseudobulges are able to launch and maintain powerful jets. High fraction of interacting sources may indicate that mergers and interaction could affect the level of nuclear activity in NLS1 galaxies. This could also explain the diversity seen in the NLS1 galaxy population as a consequence of galaxy evolution via mergers and interaction.
\end{abstract}

Revisiting narrow-line Seyfert 1 galaxies and their place in the Universe - NLS1 Padova 9-13 April 2018

Padova Botanical Garden, Italy

\footnotetext{
*Speaker.
} 


\section{Introduction}

Narrow-line Seyfert 1 galaxies (NLS1) were first described in 1985 [30] as sources exhibiting unusually narrow permitted emission lines $\left(F W H M(\mathrm{H} \beta)<2000 \mathrm{~km} \mathrm{~s}^{-1}\right)$ [15], and weak [O III] $\lambda 5007$ emission compared to the $\mathrm{H} \beta(\mathrm{F}([\mathrm{O} \mathrm{III}]) / \mathrm{F}(\mathrm{H} \beta)<3)$ [30]. In addition Fe II emission is relatively strong in the majority of NLS1 sources [30]. So far 20 NLS1 galaxies have been detected at gamma-rays (e.g., $[1,32,23]$ ), confirming that they are able to launch and maintain powerful relativistic jets.

NLS1 galaxies harbour low or intermediate mass black holes $\left(M_{\mathrm{BH}}<10^{8} M_{\odot}\right)$ [35], and seem to contradict the traditional view that only supermassive black holes $\left(M_{\mathrm{BH}}>10^{8} M_{\odot}\right)$ residing in massive ellipticals are able to launch powerful relativistic jets [24]. However, it has been proposed that the virial method widely used to estimate the black hole masses underestimates them in NLS1 galaxies due to orientation effects caused by flattened broad-line region and NLS1 sources preferentially seen face-on $[11,37]$. Some alternative methods to estimate the black hole mass support this scenario [38, 5, 3, 9], whereas others favour low black hole masses [36, 14, 44, 4].

Active galactic nuclei (AGN) have traditionally been divided to radio-loud and radio-quiet sources ${ }^{1}$, which were believed to be intrinsically different. Only $\sim 10 \%$ of NLS1 galaxies are radioloud $(R>10)$ [20], $\sim 6 \%$ radio-quiet $(R<10)$, and the remaining majority radio-silent. Recently it has been suggested that the $R$ parameter is ambiguous and generally a poor proxy for the nuclear activity in AGN $[31,16]$. This became even more evident when some radio-silent NLS1 sources were detected at $37 \mathrm{GHz}$ at several hundred mJy levels [23], indicating that they host powerful jets. Overall the NLS1 population exhibits a wide variety of properties and it seems probable that the population is heterogeneous. Yet it remains unclear which intrinsic factors explain the diversity, and how these observationally distinct subpopulations are connected.

Different host galaxies could partly explain the diversity since the properties of the host are known to affect the activity of the central engine (e.g., [43, 18]). Especially interaction can be crucial for the evolution of a galaxy since it can replenish the gas reservoirs of a galaxy, cause gas infall and subsequently trigger the nuclear activity. However, the connection between interaction and AGN activity is not yet established (e.g., [42, 7, 13, 19, 6]). Hosts of the non-jetted NLS1 galaxies are preferentially, but not exclusively, late-type galaxies. Properties indicating efficient feeding of the black hole via secular processes are commonly present. A high fraction of them show stellar bars [8, 27], nuclear dust spirals [12] and enhanced nuclear star formation [12, 39]. The fraction of interaction is significantly smaller than among the general Seyfert population $(8 \%-$ $16 \%$ vs. $20 \%-30 \%,[40,27])$. Growth mainly via secular processes is also supported by the prevalence of pseudobulges in NLS1 galaxies [29]. The hosts of jetted NLS1 galaxies are much less known, and only four individuals have been studied so far. However, different studies do not agree on the results. Elliptical, spiral and lenticular hosts have been reported, as well as undisturbed and interacting morphologies [46, 2, 25, 21, 28, 10, 9]. More extensive studies of the host galaxies of jetted NLS1 sources are needed to clarify the situation.

Increasing the number of jetted NLS1 galaxies with known host morphologies allows statistically meaningful comparison with non-jetted NLS1 sources, and offers a new perspective on,

\footnotetext{
${ }^{1}$ Radio-loudness, $R$, is defined as the ratio between radio flux density, $S_{R}$, and optical flux density, $S_{O}$, originally at $5 \mathrm{GHz}$ and in $B$-band, respectively [17].
} 
Table 1: Basic properties of the sample and $J$-band observations.

\begin{tabular}{llllll}
\hline \hline Source & $\mathrm{z}$ & $\begin{array}{l}\text { RA } \\
(\mathrm{J} 2000.0)\end{array}$ & $\begin{array}{l}\text { Dec } \\
(\mathrm{J} 2000.0)\end{array}$ & $\begin{array}{l}\text { Exp. time } \\
(\mathrm{s})\end{array}$ & $\begin{array}{l}\text { Seeing } \\
(")\end{array}$ \\
\hline SDSS J091313.73+365817.2 & 0.1073 & $09: 13: 13.72$ & $36: 58: 17.23$ & 3600 & 0.7 \\
SDSS J11934.01+533518.7 & 0.1060 & $11: 19: 34.04$ & $53: 35: 18.11$ & 1800 & 0.7 \\
SDSS J122749.14+321458.9 & 0.1368 & $12: 27: 49.15$ & $32: 14: 59.04$ & 1800 & 1.2 \\
SDSS J123220.11+495721.8 & 0.2619 & $12: 32: 20.11$ & $49: 57: 21.75$ & 3600 & 1.0 \\
SDSS J125635.89+500852.4 & 0.2453 & $12: 56: 35.87$ & $50: 08: 52.54$ & 3600 & $0.9-1.4$ \\
SDSS J133345.47+414127.7 & 0.2252 & $13: 33: 45.47$ & $41: 41: 27.66$ & 3600 & 1.1 \\
SDSS J151020.06+554722.0 & 0.1497 & $15: 10: 20.06$ & $55: 47: 22.04$ & 3600 & $1.3-1.7$ \\
SDSS J152205.41+393441.3 & 0.0766 & $15: 22: 05.41$ & $39: 34: 40.71$ & 3600 & 1.2 \\
SDSS J161259.83+421940.3 & 0.2331 & $16: 12: 59.84$ & $42: 19: 40.32$ & 3600 & 0.8 \\
\hline
\end{tabular}

for example, the heterogeneity and the intraclass evolution of the NLS1 population. It may also clarify the place of NLS1 galaxies among the young AGN. Additionally, NLS1 galaxies as young jetted AGN give new insight into the conditions necessary to launch and maintain powerful jets. Throughout the paper we assume a cosmology with $\mathrm{H}_{0}=73 \mathrm{~km} \mathrm{~s}^{-1} \mathrm{Mpc}^{-1}, \Omega_{\text {matter }}=0.27$ and $\Omega_{\text {vacuum }}=0.73[41]$.

\section{Sample, observations, and image analysis}

All nine of our sources are included in the Metsähovi NLS1 observing programme [22, 23], and seven of them have been detected at $37 \mathrm{GHz}$. Basic properties of the sample and the observations are listed in Table 1. The high resolution imaging of the NOTCam ${ }^{2}$ at the Nordic Optical Telescope was used to perform the $J$-band observations during two nights, March 3-4, 2017. The weather and seeing during the observations were variable affecting the modelling of some sources. For the point spread function (PSF) modelling we observed a bright nearby star for each source. The data was reduced using the NOTCam data reduction package ${ }^{3}$ for $\mathrm{IRAF}^{4}$ and following the standard procedure.

The 2D fitting algorithm GALFIT v3 [33] was used to perform the photometric decomposition of the images. The background sky value was estimated using IRAF and kept fixed during fitting. A PSF was used to model the AGN, and the host galaxy was modelled with an adequate number of functions, convolved with the PSF. For the bulge and bar a Sérsic profile was used, and for the disk an exponential function. The errors of the fits were estimated by fitting with $\pm 1 \sigma$ sky values, and for magnitudes an additional zeropoint estimation error was added. After sufficiently modelling the sources the radial surface brightness profiles were extracted from the observed, model, and separate component images using IRAF task ELLIPSE, which fits concentric elliptical isophotes to an image. An ellipticity/position angle (PA) versus radius plot was used as an additional diagnostic in cases when the fit indicated the presence of a bar $[45,26]$.

\footnotetext{
${ }^{2}$ The field of view is $80 " \times 80 "$, giving a scale of $0.078 " / p x$.

${ }^{3}$ http://www.not.iac.es/instruments/notcam/

${ }^{4}$ IRAF is distributed by the National Optical Astronomy Observatories, which are operated by the Association of Universities for Research in Astronomy, Inc., under cooperative agreement with the National Science Foundation.
} 
Table 2: Best fit parameters of SDSS J152205.41+393441.3. $\chi_{v}^{2}=1.03_{-0.12}^{+0.21}$.

\begin{tabular}{lllllll}
\hline \hline funct & mag & $\begin{array}{l}r_{e} \\
(\mathrm{kpc})\end{array}$ & $n$ & $\begin{array}{l}\text { axial } \\
\text { ratio }\end{array}$ & $\begin{array}{l}\text { PA } \\
\left({ }^{\circ}\right)\end{array}$ & notes \\
\hline PSF & $18.24_{-0.00}^{+0.00}$ & & & & & \\
Sérsic 1 & $16.38_{-0.07}^{+0.08}$ & $1.62_{-0.09}^{+0.12}$ & $0.84_{-0.01}^{+0.08}$ & $0.63_{-0.01}^{+0.00}$ & $-0.5_{-0.2}^{+0.7}$ & bulge \\
Sérsic 2 & $14.96_{-0.28}^{+0.38}$ & $6.14_{-0.02}^{+0.12}$ & $0.96_{-0.02}^{+0.00}$ & $0.24_{-0.04}^{+0.04}$ & $-76.5_{-0.3}^{+0.2}$ & bar \\
Expdisk & $14.30_{-0.06}^{+0.00}$ & $9.24_{-1.55}^{+2.75}$ & {$[1]$} & $0.53_{-0.03}^{+0.03}$ & $-75.9_{-0.5}^{+0.0}$ & disk \\
Sérsic 3 & $16.18_{-0.00}^{+0.03}$ & $0.51_{-0.01}^{+0.00}$ & $2.83_{-0.20}^{+0.00}$ & $0.93_{-0.03}^{+0.00}$ & $-12.4_{-1.8}^{+14.0}$ & companion \\
\hline
\end{tabular}

\section{Results: SDSS J152205.41+393441.3}

We present the modelling of one source as an example. SDSS J152205.41+393441.3 was previously classified as radio-quiet, but has been detected at $37 \mathrm{GHz}$ with the maximum flux density of $1.43 \mathrm{Jy}$. Based on the observed image (Fig. 1) it seems to be an interacting source. To ensure this we obtained the spectra of the two sources using the Asiago 1.82m Copernico telescope. The spectra confirmed that they are at the same redshift. The parameters of the best fit are given in Table 2 . Fig. 1 shows the observed, model, and residual images, and Fig. 2 the radial surface brightness profiles, and the ellipticity/PA versus radius plot. The modelling confirms that J152205.41+393441.3 is a late-type galaxy, with a pseudobulge $(n=0.84)$. The other Sérsic component resembles a bar with $n=0.96, r_{e}=6.1 \mathrm{kpc}$, an axial ratio of 0.24 . In the ellipticity/PA versus radius plot a region fitting the description of a bar is seen between $0.75-2.5$ arcsec. The bulge model is slightly extended towards the companion, explaining the rather small axial ratio. The companion galaxy is likely a dwarf elliptical. Considerable residuals confirm the disturbed morphology of the system. Especially prominent are the large structures resembling tidal tails and seemingly attached to the ends of the bar. The $\mathrm{S} / \mathrm{N}$ of these structures is $\sim 4$, suggesting they are real.
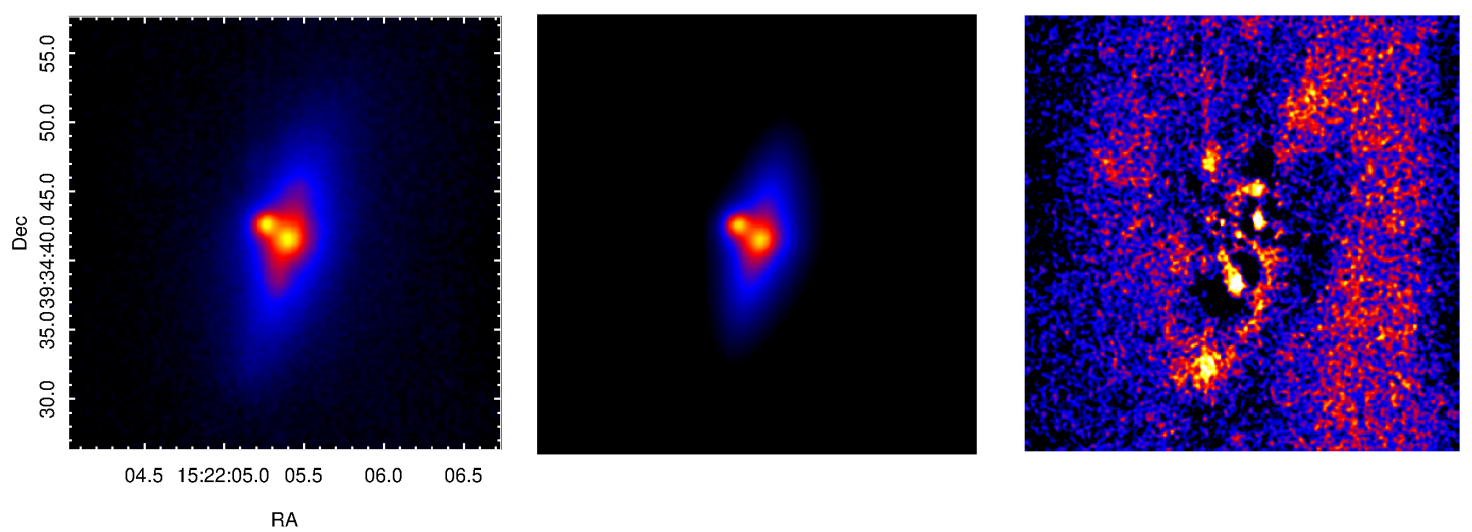

Figure 1: SDSS J152205.41+393441.3. The field of view is $23.4 " / 58.8 \mathrm{kpc}$ in all images. The NLS1 nucleus is located in the more extended, eastern, galaxy. Left panel: observed image, middle panel: model image, and right panel: residual image, smoothed over $3 \mathrm{px}$. 

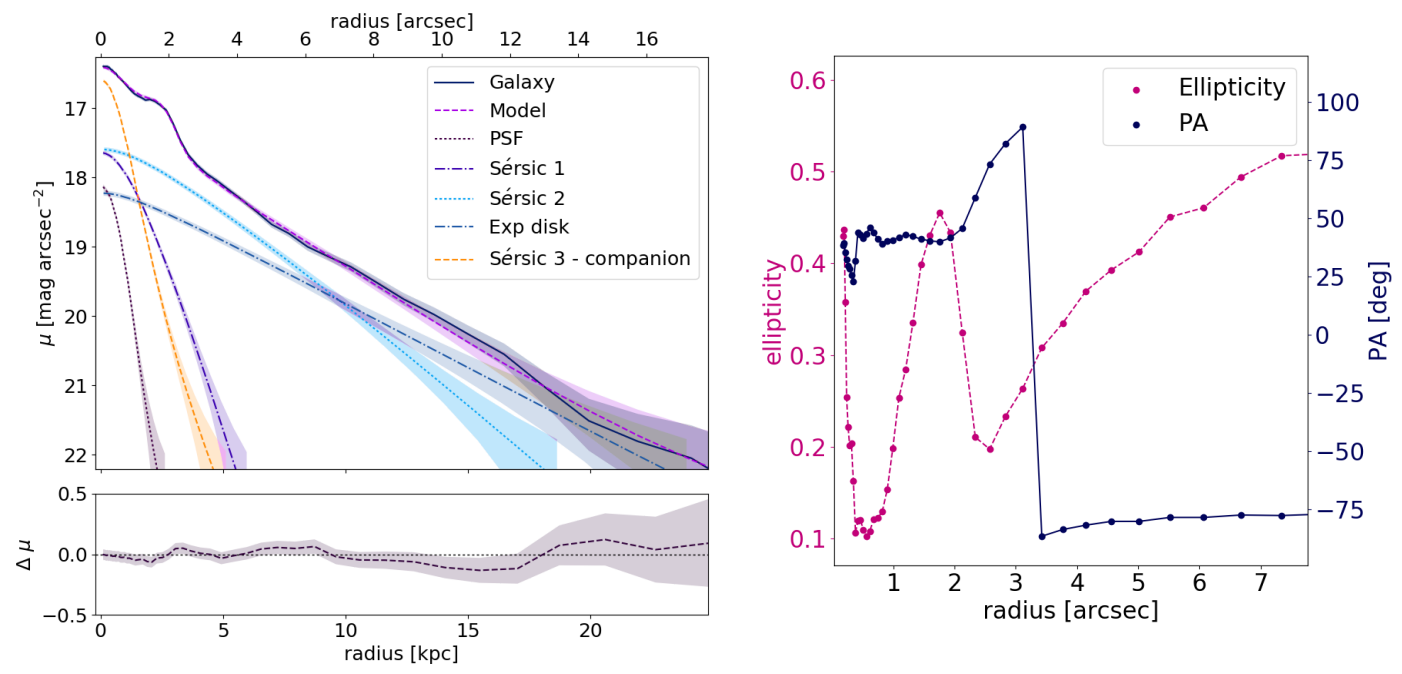

Figure 2: SDSS J152205.41+393441.3. Left panel: the observed and model radial surface brightness profiles. The shaded area describes the associated errors. Right panel: The ellipticity/PA plotted against the major axis of the isophote. Symbols and colours are explained in the plots.

\section{Discussion and conclusions}

Successful fits were achieved for five sources, three of which have been detected at $37 \mathrm{GHz}$ and can be assumed to host relativistic jets. The remaining four sources suffered a combination of poor seeing and quite high redshifts and could not be modelled reliably. The summary of the results is given in Table 3. All reliably fitted sources are late-type galaxies and harbour black holes with masses below $10^{8} M_{\odot}{ }^{5}$. Four of them host pseudobulges - the fifth does not show a distinguishable bulge - and four of them are barred. It is remarkable that none of them is an elliptical galaxy. This result, combined with other extraordinary properties of NLS1 galaxies, for example, the low black hole masses, continue to seriously challenge the traditional AGN paradigm.

Compared to the host galaxies of the general NLS1 population the sources in our sample are quite similar: disk galaxies with pseudobulges and bars, but the frequency of interaction seems to be higher. Since their other properties match those of late-type galaxies they probably have not previously undergone major mergers. Two out of three interacting sources are jetted based on $37 \mathrm{GHz}$ detections. The abundance of interaction may indicate it is indeed connected to higher levels of nuclear activity and subsequently the launching of relativistic jets in NLS1 galaxies, but the sample size needs to be increased to confirm this.

These results support the heterogeneous nature of the NLS1 population. If interaction proves to be important in determining the level of the nuclear activity the observed heterogeneity might be due to the galaxy evolution. The discovery that jetted NLS1 galaxies are found in denser largescale environments than non-jetted NLS1 galaxies supports this scenario [16] since the probability of interaction, which might lead to the launching of a jet, is naturally higher in regions with more galaxies.

\footnotetext{
${ }^{5}$ Black hole masses were estimated using the second-order moment of $\mathrm{H} \beta$ [14], which is less biased for the broadline region geometry and inclination than $\operatorname{FWHM}(\mathrm{H} \beta)[34]$.
} 
Table 3: Summary of the results.

\begin{tabular}{llllll}
\hline \hline source & morphology & components & jets & $\begin{array}{l}\log M_{\mathrm{BH}} \\
\left(M_{\odot}\right)\end{array}$ & notes \\
\hline $\mathrm{J} 091313.73+365817.2$ & spiral & $\mathrm{PB}$, bar, disk & & 6.9 & enhanced SF \\
$\mathrm{J} 111934.01+533518.7$ & spiral & $\mathrm{PB}$ & & 6.3 & $\begin{array}{l}\text { interacting, RL } \\
\text { enhanced SF }\end{array}$ \\
$\mathrm{J} 122749.14+321458.9$ & unclear & & yes & 7.2 & \\
$\mathrm{~J} 123220.11+495721.8$ & unclear & & yes & 7.5 & \\
$\mathrm{~J} 125635.89+500852.4$ & unclear & & yes & 6.7 & \\
$\mathrm{~J} 133345.47+414127.7$ & unclear & & yes & 7.8 & starburst \\
$\mathrm{J} 151020.06+554722.0$ & spiral & bar, disk & yes & 6.7 & \\
$\mathrm{~J} 152205.41+393441.3$ & spiral & PB, bar, disk & yes & 6.2 & interacting \\
$\mathrm{J} 161259.83+421940.3$ & spiral & PB, bar & yes & 6.8 & disturbed, starburst \\
Col. 1 gives the source name, Col. 2 lists the probable morphology of the host galaxy and Col. 3 gives the components \\
of the model; PB denotes pseudobulge. In Col. 4 a source is marked if it has been detected at 37 GHz. The black hole \\
\multicolumn{5}{c}{ mass in given in Col. 5. Col. 6 gives additional notes of the source; SF denotes star formation. }
\end{tabular}

\section{Acknowledgements}

This conference has been organized with the support of the Department of Physics and Astronomy "Galileo Galilei", the University of Padova, the National Institute of Astrophysics INAF, the Padova Planetarium, and the RadioNet consortium. RadioNet has received funding from the European Union's Horizon 2020 research and innovation programme under grant agreement No 730562.

\section{References}

[1] Abdo, A. A., Ackermann, M., Ajello, M., et al. 2009, ApJ, 699, 976

[2] Antón, S., Browne, I. W. A., \& Marchã, M. J. 2008, A\&A, 490, 583

[3] Baldi, R. D., Capetti, A., Robinson, A., Laor, A., \& Behar, E. 2016, MNRAS, 458, L69

[4] Berton, M., Congiu, E., Järvelä, E., et al. 2018, ArXiv:1801.03519

[5] Calderone, G., Ghisellini, G., Colpi, M., \& Dotti, M. 2013, MNRAS, 431, 210

[6] Chiaberge, M., Gilli, R., Lotz, J. M., \& Norman, C. 2015, ApJ, 806, 147

[7] Corbin, M. R. 2000, ApJL, 536, L73

[8] Crenshaw, D. M., Kraemer, S. B., \& Gabel, J. R. 2003, AJ, 126, 1690

[9] D’Ammando, F., Acosta-Pulido, J. A., Capetti, A., et al. 2018, MNRAS, 478, L66

[10] D’Ammando, F., Acosta-Pulido, J. A., Capetti, A., et al. 2017, MNRAS, 469, L11

[11] Decarli, R., Dotti, M., Fontana, M., \& Haardt, F. 2008, MNRAS, 386, L15

[12] Deo, R. P., Crenshaw, D. M., \& Kraemer, S. B. 2006, AJ, 132, 321

[13] Ellison, S. L., Patton, D. R., Mendel, J. T., \& Scudder, J. M. 2011, MNRAS, 418, 2043

[14] Foschini, L., Berton, M., Caccianiga, A., et al. 2015, A\&A, 575, A13

[15] Goodrich, R. W. 1989, ApJ, 342, 224 
[16] Järvelä, E., Lähteenmäki, A., Lietzen, H., et al. 2017, A\&A, 606, A9

[17] Kellermann, K. I., Sramek, R., Schmidt, M., Shaffer, D. B., \& Green, R. 1989, AJ, 98, 1195

[18] King, A. \& Pounds, K. 2015, ARA\&A, 53, 115

[19] Kocevski, D. D., Faber, S. M., Mozena, M., et al. 2012, ApJ, 744, 148

[20] Komossa, S., Voges, W., Xu, D., et al. 2006, AJ, 132, 531

[21] Kotilainen, J. K., León-Tavares, J., Olguín-Iglesias, A., et al. 2016, ApJ, 832, 157

[22] Lähteenmäki, A., Järvelä, E., Hovatta, T., et al. 2017, A\&A, 603, A100

[23] Lähteenmäki, A., Järvelä, E., Ramakrishnan, V., et al. 2018, A\&A, 614, L1

[24] Laor, A. 2000, ApJL, 543, L111

[25] León Tavares, J., Kotilainen, J., Chavushyan, V., et al. 2014, ApJ, 795, 58

[26] Menéndez-Delmestre, K., Sheth, K., Schinnerer, E., Jarrett, T. H., \& Scoville, N. Z. 2007, ApJ, 657, 790

[27] Ohta, K., Aoki, K., Kawaguchi, T., \& Kiuchi, G. 2007, ApJS, 169, 1

[28] Olguín-Iglesias, A., Kotilainen, J. K., León Tavares, J., Chavushyan, V., \& Añorve, C. 2017, MNRAS, 467, 3712

[29] Orban de Xivry, G., Davies, R., Schartmann, M., et al. 2011, MNRAS, 417, 2721

[30] Osterbrock, D. E. \& Pogge, R. W. 1985, ApJ, 297, 166

[31] Padovani, P. 2016, A\&ARv, 24, 13

[32] Paliya, V. S., Ajello, M., Rakshit, S., et al. 2018, ApJL, 853, L2

[33] Peng, C. Y., Ho, L. C., Impey, C. D., \& Rix, H.-W. 2010, AJ, 139, 2097

[34] Peterson, B. M. 2011, ArXiv:1109.4181

[35] Peterson, B. M., McHardy, I. M., Wilkes, B. J., et al. 2000, ApJ, 542, 161

[36] Rafter, S. E., Kaspi, S., Chelouche, D., et al. 2013, ApJ, 773, 24

[37] Rakshit, S., Stalin, C. S., Chand, H., \& Zhang, X.-G. 2017, ApJS, 229, 39

[38] Ryan, C. J., De Robertis, M. M., Virani, S., Laor, A., \& Dawson, P. C. 2007, ApJ, 654, 799

[39] Sani, E., Lutz, D., Risaliti, G., et al. 2010, MNRAS, 403, 1246

[40] Schmitt, H. R., Antonucci, R. R. J., Ulvestad, J. S., et al. 2001, ApJ, 555, 663

[41] Spergel, D. N., Bean, R., Doré, O., et al. 2007, ApJs, 170, 377

[42] Taniguchi, Y. 1999, ApJ, 524, 65

[43] van de Ven, G. \& Fathi, K. 2010, ApJ, 723, 767

[44] Wang, F., Du, P., Hu, C., et al. 2016, ApJ, 824, 149

[45] Wozniak, H., Friedli, D., Martinet, L., Martin, P., \& Bratschi, P. 1995, A\&AS, 111, 115

[46] Zhou, H., Wang, T., Yuan, W., et al. 2007, ApJL, 658, L13 